\title{
Cartographie De La Dynamique Historique Du Trait De Côte Des Plages De Guédiawaye Et Malika (Dakar, Sénégal)
}

\author{
Papa Sagne \\ Kader $\mathbf{B a}$
}

Laboratoire de Biostratigraphie-Sédimentologie, Département de Géologie,

Université Cheikh Anta Diop de Dakar, Sénégal

Laboratoire de Télédétection Appliquée, Institut des Sciences de la Terre,

Université Cheikh Anta Diop de Dakar, Sénégal

\section{Boubacar Fall}

Laboratoire de Biostratigraphie-Sédimentologie, Département de Géologie,

Université Cheikh Anta Diop de Dakar, Sénégal

\section{Jean Paul Marcel Youm}

Laboratoire de Biostratigraphie-Sédimentologie, Département de Géologie,

Université Cheikh Anta Diop de Dakar, Sénégal.

Laboratoire de Télédétection Appliquée, Institut des Sciences de la Terre,

Université Cheikh Anta Diop de Dakar, Sénégal

\section{Gayane Faye}

Laboratoire de Télédétection Appliquée, Institut des Sciences de la Terre,

Université Cheikh Anta Diop de Dakar, Sénégal

\section{Jean Pierre Gane Sarr \\ El Hadji Sow}

Laboratoire de Biostratigraphie-Sédimentologie, Département de Géologie,

Université Cheikh Anta Diop de Dakar, Sénégal

Doi:10.19044/esj.2021.v17n25p214

Submitted: 11 March 2021

Accepted: 21 June 2021

Published: 31 July 2021
Copyright 2021 Author(s)

Under Creative Commons BY-NC-ND 4.0 OPEN ACCESS

Cite As:

Sagne P., Ba K., Fall B., Youm J.P.M., Faye G., Sarr J.P.G. \& Sow E.H. (2021). Cartographie De La Dynamique Historique Du Trait De Côte Des Plages De Guédiawaye Et Malika (Dakar, Sénégal). European Scientific Journal, ESJ, 17(25), 214.

https://doi.org/10.19044/esj.2021.v17n25p214

\section{Résumé}

L'érosion côtière est une des causes de la disparition des plages, du recul des falaises et de la perte de terrains à valeur socio-économique et 
écologique. La compréhension de la dynamique côtière est donc primordiale avant d'entreprendre toute politique d'aménagement du littoral.

Cette étude se propose de retracer la dynamique historique du trait de côte de la zone de Malibu à Malika au Nord de Dakar qui subit actuellement une forte pression humaine avec l'extension de la Voie de Dégagement Nord. Elle est basée sur l'analyse de données de télédétection au moyen du logiciel ArcGIS 10.4 et de l'extension DSAS 4.3 (Digital Shoreline Analysis System). Les résultats obtenus laissent apparaitre, entre 1942 et 2011, une évolution du trait de côte en faveur d'une érosion avec une vitesse moyenne de $-0,15 \mathrm{~m} / \mathrm{an}$. Cette évolution, qui n'est pas uniforme d'un secteur à un autre mais aussi d'une période à une autre, serait essentiellement due à l'action des agents hydrodynamiques qui gouvernent les mouvements sédimentaires.

Néanmoins, la pression humaine commence à se faire sentir avec l'extension de la Voie de Dégagement Nord, les extractions de sables et la coupe des filaos au profit des constructions humaines dont les conséquences sur la dynamique côtière peuvent être alarmantes.

Mots clés : Littoral, Dakar, SIG, Télédétection, Érosion 


\title{
Mapping Of The Historical Coastline Dynamics Of Guediawaye And Malika Beaches (Dakar, Senegal)
}

\section{Papa Sagne \\ Kader Ba}

Laboratoire de Biostratigraphie-Sédimentologie, Département de Géologie,

Université Cheikh Anta Diop de Dakar, Sénégal

Laboratoire de Télédétection Appliquée, Institut des Sciences de la Terre,

Université Cheikh Anta Diop de Dakar, Sénégal

\section{Boubacar Fall}

Laboratoire de Biostratigraphie-Sédimentologie, Département de Géologie,

Université Cheikh Anta Diop de Dakar, Sénégal

\section{Jean Paul Marcel Youm}

Laboratoire de Biostratigraphie-Sédimentologie, Département de Géologie,

Université Cheikh Anta Diop de Dakar, Sénégal.

Laboratoire de Télédétection Appliquée, Institut des Sciences de la Terre,

Université Cheikh Anta Diop de Dakar, Sénégal

Gayane Faye

Laboratoire de Télédétection Appliquée, Institut des Sciences de la Terre,

Université Cheikh Anta Diop de Dakar, Sénégal

\section{Jean Pierre Gane Sarr \\ EI Hadji Sow}

Laboratoire de Biostratigraphie-Sédimentologie, Département de Géologie,

Université Cheikh Anta Diop de Dakar, Sénégal

\begin{abstract}
Coastal erosion is one of the causes of the disappearance of beaches, retreat of cliffs and loss of land with socio-economic and ecological value. Understanding coastal dynamics is therefore essential before undertaking any coastal development policy.

This study aims to retrace the historical dynamics of the coastline from the Malibu to Malika area located in the north of Dakar which is currently under strong human pressure with the extension of the Northern Clearance Way. It is based on the analysis of remote sensing data using ArcGis 10.4 and the DSAS 4.3 extension (Digital Shoreline Analysis System).

The results obtained show, between 1942 and 2011, an evolution of the coastline in favor of erosion with an average speed of $-0.15 \mathrm{~m} /$ year. This evolution, which is not uniform from a sector to another, but also from one period to another, is essentially due to the hydrodynamic agents which govern sediment movements.

However, human pressure is starting to be felt with the extension of the Northern Clearance Way, sand extraction and the cutting of casuarinas for
\end{abstract}


human constructions benefit. The consequences of such practices on coastal dynamics can be alarming.

Keywords: Coast, Dakar, GIS, Remote Sensing, Erosion

\section{Introduction}

Situé sur la pointe extrême occidentale du continent africain, le Sénégal est un pays côtier avec une façade littorale de 706,72 km de long environ (Diaw, 1984) où se concentrent 58,7 \% de la population avec un taux de croissance annuel estimé à 2,7 \% entre 2002-2013 (ANSD, 2014). Selon le rapport du CSE sur l'état de l'environnement (2010), cette façade littorale sénégalaise traverse six (6) régions administratives (Saint Louis, Louga, Thiès, Dakar, Fatick, et Ziguinchor) où se trouvent des villes dont les activités économiques contribuent à hauteur de $68 \%$ du produit intérieur brut du pays. En effet, cette zone abrite $90 \%$ des industries et l'essentiel de l'activité touristique qui est principalement balnéaire. La pêche côtière y est également très développée. Par ailleurs, elle fournit jusqu'environ $70 \%$ des fruits et légumes consommés, à travers l'agriculture péri-urbaine (CSE, 2010).

Malgré ces potentialités importantes, cette zone est confrontée à de nombreux défis dont l'érosion côtière (Balle et al., 2021) qui est reconnue comme étant l'un des risques naturels majeurs dans le Plan d'Action National d'Adaptation du Sénégal (PANA, 2006). L'érosion côtière est un phénomène naturel engendré par la conjonction d'un certain nombre de facteurs dynamiques tels que les houles, surtout celles exceptionnelles (Suanez et Cariolet, 2010 ; Stéphanian et al., 2011 ; Konan et al., 2016), les vagues et la marée (Bird, 1985 ; Ibe et Quélennec 1989 ; Paskoff, 1993 ; Niang-Diop, 1995 ; Pinot, 1998 et Mugica et al., 2008 ; CSE, 2010). Cependant, ce phénomène est actuellement exacerbé dans beaucoup de zones par les changements climatiques et surtout les actions anthropiques qui perturbent parfois l'équilibre dynamique des milieux littoraux (Daniel et Hubaud, 1996). La compréhension de la dynamique côtière est donc primordiale dans l'aménagement du littoral. Elle constitue une préoccupation commune des géomorphologues et des gestionnaires des espaces côtiers (Hénaff et al., 2015). Elle est souvent basée sur des méthodes topographiques (Young et al., 2010 ; Thior et al., 2019), sédimentologiques et cartographiques (Faye, 2010 ; Emmanuel, 2017).

Les méthodes cartographiques permettent le plus souvent de retracer les fluctuations ou cinématiques du trait de côte sur plusieurs décennies. Elles sont d'habitude axées sur l'analyse de données de télédétection par l'utilisation des systèmes d'information géographique (SIG) (Faye 2010, Emmanuel, 2017). Ces méthodes ont été initialement utilisées dans un but purement théorique (Niang-Diop, 1995), mais elles ont connu à partir de la fin 
des années 80 de plus en plus d'applications pratiques, notamment pour l'évaluation des impacts des travaux de génie côtier, la détermination des volumes de sables nécessaires pour l'alimentation artificielle des plages et l'estimation des changements futurs de la ligne de rivage (Niang-Diop, 1995). Elles sont de ce fait, devenues un élément indispensable à la définition des politiques d'aménagement du littoral (Grenier et Dubois, 1990).

Cette présente étude, basée sur le traitement d'images satellitaires et de photographies aériennes multi-dates au moyen du logiciel « ArcGIS 10.4 » et de l'extension DSAS 4.3 (Digital Shoreline Analysis System), a pour objectif de retracer la dynamique du trait de côte de la zone de Malibu à Malika au Nord de Dakar entre 1942 et 2011 soit 69 ans d'évolution.

\section{Cadre Experimental}

\section{Présentation de la zone d'étude}

Cette étude concerne le littoral nord de Dakar plus particulièrement les communes de Golf Sud, Ndiarème Limamoulaye, Sam Notaire, Wakhinane Nimzatt situées dans le département de Guédiawaye et celles de Yeumbeul Nord et Malika dans le département de Pikine (figure 1).

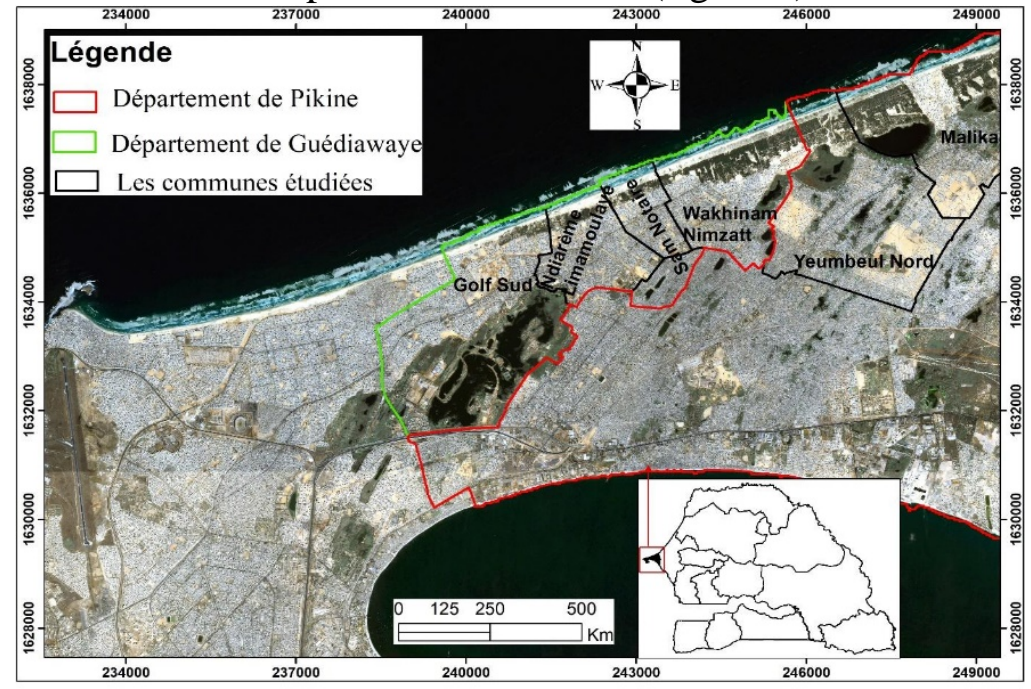

Figure 1. Situation de la zone d'étude

Pikine est le département le plus peuplé de la région de Dakar voire du Sénégal avec environ 1170791 habitants, soit 37,32 \% de la population de Dakar (SRSD, 2015). Il est suivi par le département de Dakar qui abrite une population d'environ 1146053 habitants soit 36,53 \% de la population de la région de Dakar (figure 2). Ceux de Rufisque et Guédiawaye arrivent très loin derrière, avec respectivement 490694 et 329659 habitants (SRSD, 2015). 


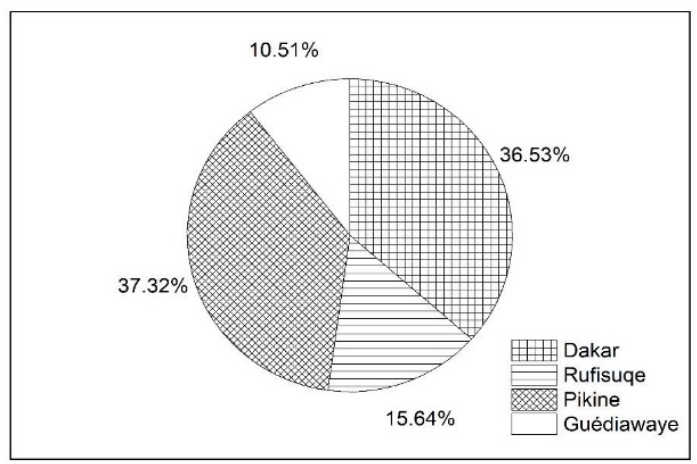

Figure 2. Répartition spatiale de la population de Dakar selon les départements (SRSD, 2015)

La commune de Yeumbeul Nord est la deuxième commune la plus peuplée du département de Pikine après celle de Keur Massar (figure 3) avec une population de 168379 habitants (SRSD, 2015). Dans le département de Guédiawaye, la commune la plus peuplée est celle de Golf Sud avec 92325 habitants. Elle est suivie de très près par la commune de Wakhinane Nimzatt qui abrite 89721 habitants du département. La commune d'arrondissement de Ndiarème Limamoulaye est la moins peuplée de Guédiawaye avec une population de 35171 habitants (SRSD, 2015).

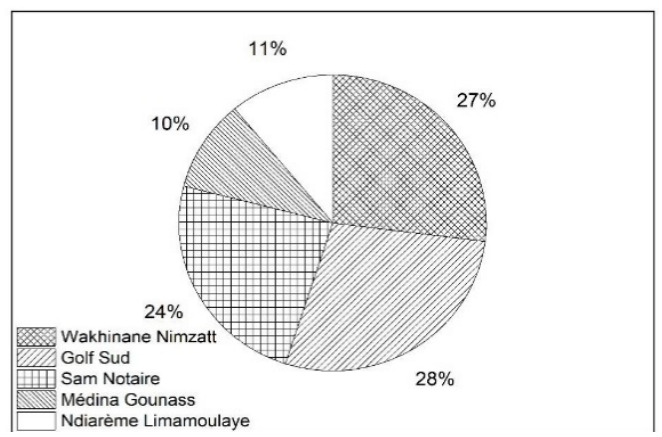

Figure 3. Répartition spatiale de la population du département de Guédiawaye (SRSD, 2015)

Il est important de noter que la population des départements de Pikine et Guédiawaye qui couvrent notre zone d'étude risque d'augmenter de manière plus considérable dans les années à venir en raison de l'extension de la Voie de Dégagement Nord (photo 1) qui permet quelque part de désengorger la banlieue dakaroise en facilitant la libre circulation des personnes et des biens au Nord de Dakar. 


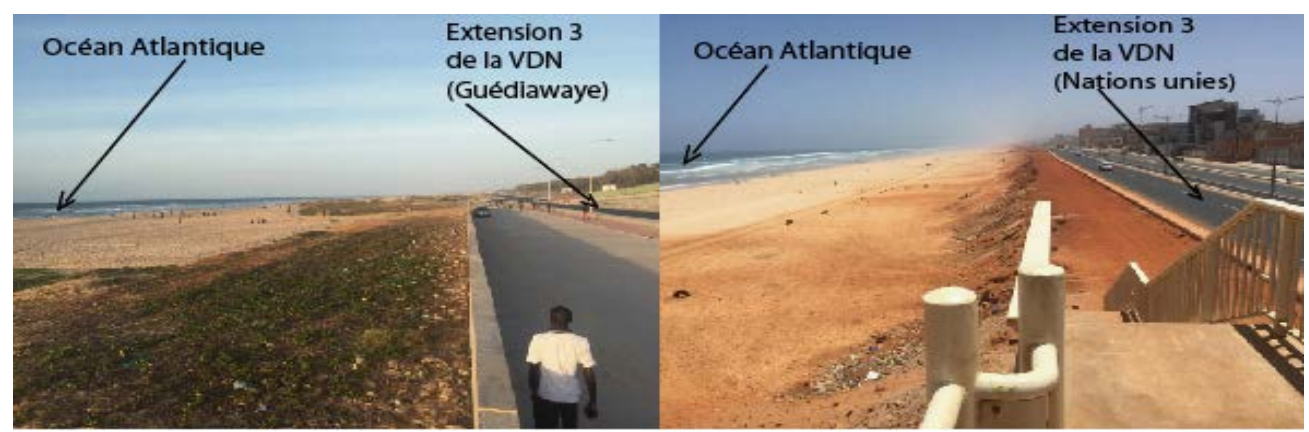

Photo 1. l'extension 3 de la Voix de Dégagement Nord

Cette forte croissance démographique pourrait, en plus de la Voie de Dégagement Nord, entrainer l'occupation de toute la bande côtière nord de Dakar occupée par des filaos qui permettent de fixer les dunes datées du Quaternaire (Roger et al, 2009). En effet, c'est la végétation littorale, représentée dans notre zone par les filaos (Casuarina equisetifolia) qui piège et stabilise le sable en mouvement pour former les dunes littorales qui sont d'une grande importance pour la stabilité du littoral (Paskoff, 1993 ; Ibe et Quélennec, 1989). Ainsi, toute destruction de ces filaos côtiers pour faire place à des constructions comme ce qui se passe actuellement dans la zone d'étude (photo 2), pourrait, sur le long terme, entrainer une disparition des dunes et la suppression des leurs rôles de protection naturelle des zones côtières (Ibe et Quélennec, 1989).

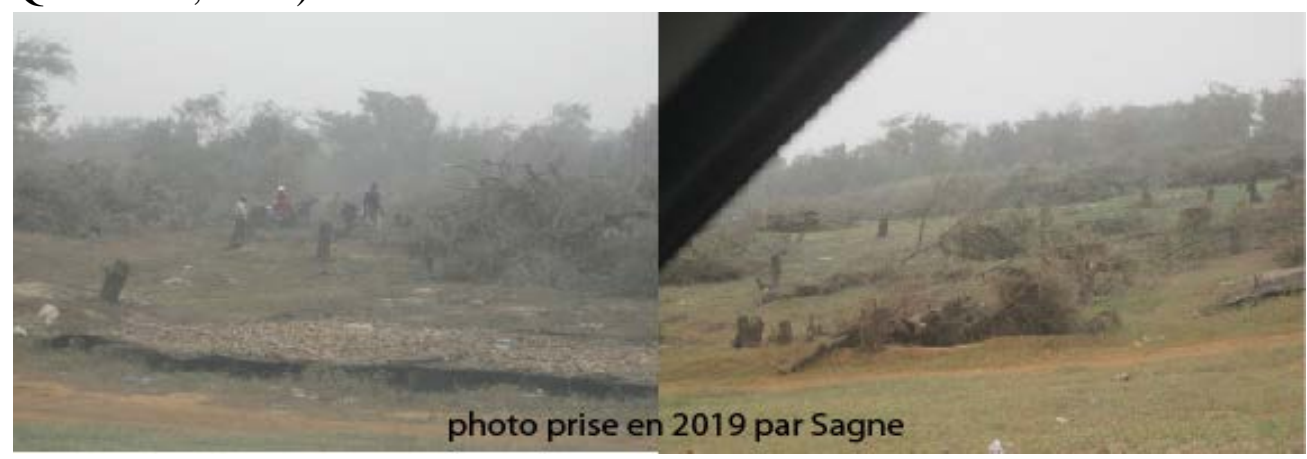

Photo 2. Coupe de filaos dans la zone de Malika

Sur les côtes sénégalaises, la marée est de type semi-diurne avec un marnage variant entre 0,5 et $0,6 \mathrm{~m}$ lors des marées de mortes eaux et entre 1,2 et 1,6 m pendant les marées de vives eaux (Niang-Diop, 1995). Par ailleurs, en plus du faible marnage (inférieur à $2 \mathrm{~m}$ ), qui lui confère le caractère de côte micro-tidale, les vitesses des courants de marées inférieures à $0,15 \mathrm{~m} / \mathrm{s}$ indiquent une faible influence de la marée sur les mouvements de ce littoral (Fall, 2004 ; Ndour, 2015 ; Stéphanain et al,. 2011).

Compte tenu de ce qui précède, les houles du Nord-Ouest auxquelles est exposée la côte nord, constituent les principaux agents responsables de la 
dynamique morphologique et sédimentaire sur cette partie du littoral de Dakar (Niang-Diop, 1995, Ndour, 2015).

\section{Description de la méthodologie}

Il existe un certain nombre de méthodes d'étude de l'évolution des littoraux (Moore, 2000 ; Le Berre et al., 2016) tant pour l'analyse des documents que pour l'exploitation des résultats. De ce fait, deux chercheurs travaillant dans la même zone peuvent obtenir des résultats différents (Crowell et Buckley, 1993). Ainsi, le choix d'une méthode adéquate dépendant des objectifs de l'étude, de la nature de la côte, de la base de données disponible et des outils de mesure est fondamental. L'utilisation de la photographie aérienne et des images satellitaires de haute résolution est bien adaptée à l'analyse de la cinématique des littoraux meubles à l'échelle de plusieurs décennies car elles offrent une grande précision (Suanez et Simon, 1997 ; Durand, 2000). De ce fait, les principales données utilisées pour la présente étude de la cinématique historique de la côte sableuse nord de Dakar sont constituées de photographies aériennes datant de 1942, 1966, 1978, 1997 et d'une image satellitaire de type Alos/Palsar (Advanced Land Observation Satellite/Phased Array type L-band Synthetic Aperture Radar) du satellite Japonais, acquise en 2011. Quelques caractéristiques concernant ces données sont consignées dans le tableau 1.

Tableau 1. Caractéristiques des données numériques utilisées

\begin{tabular}{|c|c|c|c|c|}
\hline Données & \multicolumn{2}{|c|}{ Référence de la mission } & Echelle & $\begin{array}{c}\text { Date de } \\
\text { prise de vue }\end{array}$ \\
\hline \multirow[t]{4}{*}{$\begin{array}{l}\text { Photos } \\
\text { aériennes }\end{array}$} & \multicolumn{2}{|c|}{$\begin{array}{l}\text { Mission de reconnaissance de l'armée de } \\
\text { l'air américaine }\end{array}$} & $1 / 20000$ & 4 avril 1942 \\
\hline & \multicolumn{2}{|c|}{ Mission Satellite American corona } & $1 / 20000$ & 19 février 1966 \\
\hline & \multicolumn{2}{|c|}{ IGN 1978} & $1 / 60000$ & 1978 \\
\hline & \multicolumn{2}{|c|}{ IGN 12-01-1997 } & $1 / 20000$ & 12 janvier 1997 \\
\hline \multirow[t]{2}{*}{ Alos/Palsar } & Mode & Polarisation & $\begin{array}{l}\text { Résoluti } \\
\text { on }\end{array}$ & Date \\
\hline & Ascendant & $\mathrm{HH}$ & $10 \mathrm{~m}$ & 2011 \\
\hline
\end{tabular}

Ces données ont subi un géoréférencement puis sont utilisées pour la réalisation des cartes d'évolution du trait de côte et celles de l'occupation du sol du littoral nord de Dakar.

\section{Géoréférencement}

La reproduction conforme n'est pas de règle en télédétection (Ndour, 2015). En effet, lors d'une prise de vue, les images satellitaires ainsi que les photographies aériennes subissent plusieurs déformations causées par plusieurs facteurs tels que les variations en roulis, tangage, lacet en altitude, l'angle de prise de vue (Dolan et al., 1978 ; Crowell et al., 1991 ; Thieler et Danforth, 1994 ; Moore, 2000 ; Provencher et Dubois, 2007 ; Bâ, 2013 ; Mall, 
2016). Elles ne peuvent donc être directement utilisées dans une étude d'évolution historique de la ligne de rivage du fait que certains points occupent sur les images ou photos des positions différentes de celles qu'ils occupent réellement sur le terrain. L'objectif du géoréférencement est donc, d'une part, de rectifier les déformations de la prise de vue ou erreurs géométriques, et d'autre part, de pouvoir représenter l'image à rectifier dans un système de coordonnées. Dans cette étude, le système géodésique WGS_1984 et la projection UTM fuseau 28N ont été utilisés pour projeter ces données.

La correction géométrique ou géoréférencement des données a été effectuée avec le logiciel Envi 4.2 en utilisant la méthode « image à image " ; l'image de référence étant celle du satellite Spot 5 de résolution spatiale 2,5 m prise en octobre 2006. Cet exercice nécessite l'établissement des points de contrôle ou amers (ou GCP : Ground Control Point ou Points de contrôle au Sol) qui sont des points permanents et stables dont les coordonnées géographiques (longitude, latitude et altitude) ou UTM (X, Y et Z) sont connues au sol. Ces points de control au sol sont choisis suffisamment fixes comme les carrefours, les angles de maisons, d'usines, les croisements de route etc. Ils peuvent être obtenus sur le terrain par des levés GPS ou directement sur les documents de référence. Dans notre étude, ces points sont obtenus sur l'image de référence (figure 4). Ils sont bien répartis sur les images et sont assez nombreux (17 points) avec des erreurs de correction géométrique inférieures à un demi pixel $(0,5)$ (RMS error maximum étant de 0,15 pour 17 points de contrôle). La rectification permet une superposition satisfaisante des images avant l'extraction manuelle des lignes de référence à comparer.

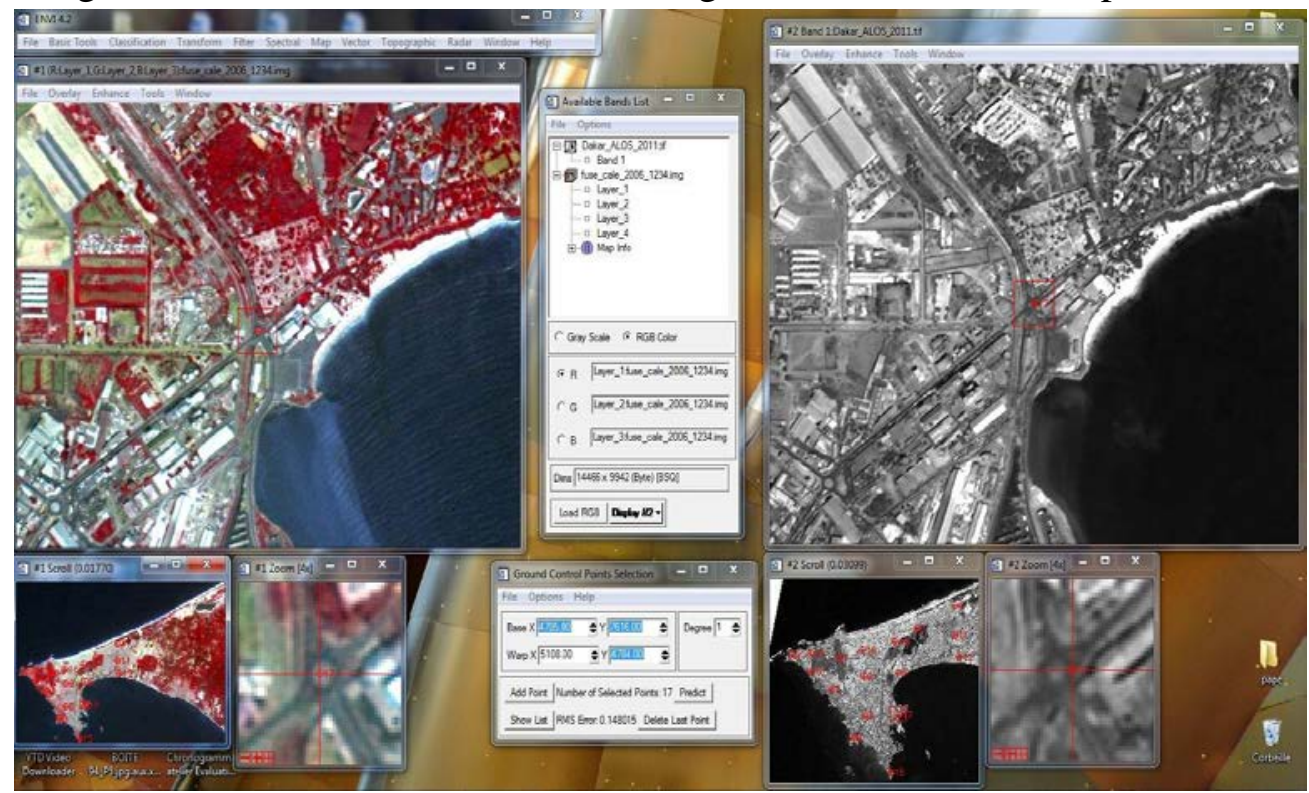

Figure 4. Répartition des points sur les images lors du géoréférencement et l'erreur moyenne quadratique ou (RMS) obtenue 


\section{Choix d'un indicateur du trait de côte}

Le littoral, situé à l'interface entre la terre, la mer et l'atmosphère, est un milieu très dynamique. Pour mieux caractériser son évolution, il est nécessaire de définir des indicateurs. Ces indicateurs peuvent être regroupés sous le concept de «trait de côte ». Dans les faits, la définition de ce concept de trait de côte encore appelé ligne de rivage dans les zones littorales de types micro-tidales (Suanez et Simon, 1997 ; Durand, 1998 ; Durand, 2000 ; Sabatier et Suanez, 2003) comme celles du Sénégal est tout à fait délicate, même problématique et sujette à controverse. Les définitions du trait de côte et les méthodes d'analyse dépendent essentiellement du type de côte (Shoshany et Degani, 1992), mais également des données disponibles et des outils de traitement des données à utiliser. Il est possible de distinguer : les lignes de rivage à référence géomorphologique, celles de végétation et les lignes instantanées de rivage et les limites d'humectation (Faye, 2010 ; Juigner et al., 2012).

Dans cette étude, c'est la limite supérieure d'humectation de l'estran qui est utilisée comme ligne de rivage (ou trait de côte) car elle est visible, continue et facilement reconnaissable sur toutes les données utilisées (figure 5). Dans ce cas, elle est la ligne qui sépare les parties mouillées qui apparaissent gris foncé et les parties sèches qui apparaissent gris clair à blanc (Dolan et al., 1978 ; Dolan et al.,1980 ; Crowell et al., 1991 ; Douglas et Crowell, 2000). En plus, sur les littoraux micro-tidaux où le marnage est relativement négligeable comme ceux du Sénégal, elle atténue l'influence de la marée au moment des prises de données (Suanez et Simon, 1997 ; Durand, 1998 ; Durand, 2000 ; Sabatier et Suanez, 2003 ; Ndour, 2015). Cependant, pour mieux atténuer les erreurs liées à la marée, il est recommandé d'utiliser des données prises à des heures de marées identiques (Ndour, 2015).

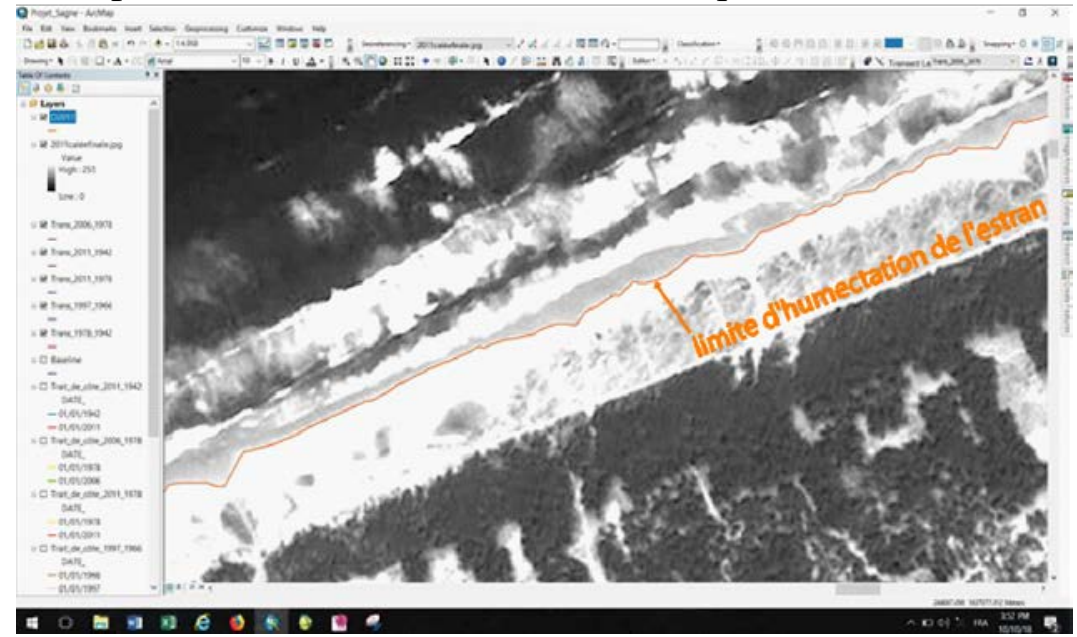

Figure 5. Limite d’humectation de la ligne de rivage sur fond photo aérienne de 1997 


\section{Méthodes statistiques d'étude de la mobilité du trait de côte}

L'analyse s'appuie sur l'utilisation des logiciels ArcGIS 10.4 et l'extension en accès libre DSAS 4.3 (Digital Shoreline Analysis System) (Thieler et al., 2009), afin de comparer l'évolution de la position du trait de côte à différentes dates. L'extension DSAS permet de décrire de manière statistique et par période, la dynamique côtière (érosion, accrétion, stabilité) (Thieler et al., 2009). Le principe consiste d'abord à une mise en forme rigoureuse des données dans une Géodatabase personnelle, ensuite à numériser pour chaque image ou photo, la ligne de rivage puis à définir une ligne de base (Baseline). Celle-ci, placée en zone mer (offshore) est parallèle aux différentes lignes de rivage. L'extension DSAS génère automatiquement, en fonction du pas choisi (en mètre), un ensemble de transects perpendiculaires aux différents traits de côte au droit de la ligne de base déterminée par l'utilisateur (figure 6). La distance entre les transects (espacement des transects ou encore pas entre les transects) est définie dans l'interface de l'extension par l'utilisateur. Elle dépend quelque part de la longueur et de la forme du linéaire côtier que l'on étudie mais surtout de la précision des résultats que l'on veut obtenir. En effet, les résultats reflètent plus la réalité du terrain quand l'espacement entre les transects est faible. Dans notre cas, cet espacement est de $50 \mathrm{~m}$ pour un segment linéaire d'un peu plus de $8 \mathrm{Km}$. Pour chaque transect, le DSAS calcule automatiquement la distance entre la ligne de base fictive et la position des traits de côte.

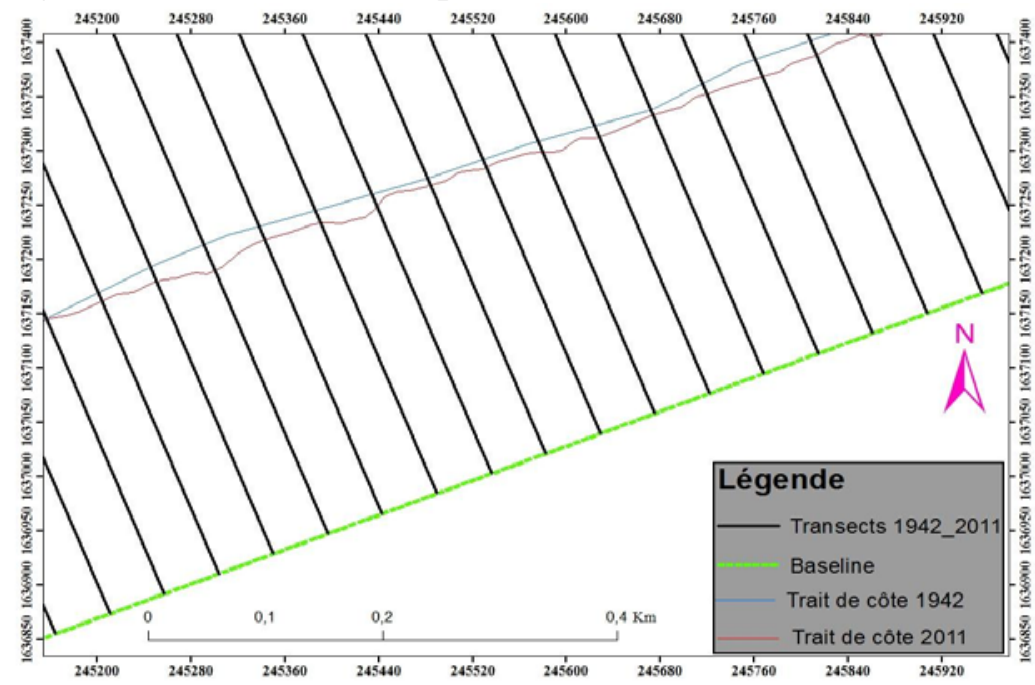

Figure 6.Transects générés par le DSAS pour la mesure de l'évolution du trait de côte

Les méthodes statistiques d'analyse quantitative de la mobilité du linéaire côtier utilisent des moyennes ou des régressions pour modéliser au mieux les tendances évolutives du littoral. 
Deux méthodes sont utilisées : la méthode des points extrêmes (End point rates) et la régression linéaire simple (Linear Regression Rate-ofchange).

- La méthode des points extrêmes (EPR) n’utilise que deux positions connues de la ligne de référence (la position la plus ancienne et la plus récente). La distance (en mètre) mesurée entre ces deux emplacements extrêmes du trait de côte durant la période d'étude est divisée par le nombre d'années écoulées pour obtenir la vitesse d'évolution annuelle du littoral. Exprimé en $\mathrm{m} / \mathrm{an}$, c'est cet attribut que l'on retrouve dans le modèle cartographique, sous le libellé : moyenne annuelle. L'avantage de cette méthode réside dans sa simplicité. Toutefois, lorsque l'on dispose de positions intermédiaires entre les deux dates extrêmes de la période d'étude, les taux estimés par cette technique n’intégreront pas les éventuelles variations temporelles dans l'évolution du littoral (accélération, ralentissement ou inversion de tendance). L'EPR convient bien lorsque l'on ne dispose que de deux traits de côte pour évaluer la cinématique littorale (Thieler et al., 2005).

- Dans le cas de la régression linéaire simple (LRR), les mesures de distances entre la ligne de référence et les positions successives du trait de côte sont utilisées pour calculer, par les moindres carrés, une droite de régression linéaire. Celle-ci est ajustée aux différents emplacements de la ligne de référence. La pente de la droite de régression correspond au taux d'évolution. La régression linéaire est la méthode la plus robuste pour estimer les tendances historiques de l'évolution du trait de côte (Dolan et al., 1991 ; Fenester et al., 1993).

\section{Estimation des erreurs ou incertitudes}

Les études d'évolution historique de la ligne de rivage souffrent de plusieurs erreurs ou incertitudes (Fletcher et al., 2003 ; Juigner, 2012). Ces erreurs peuvent avoir comme source la précision des documents de référence, la précision du repérage des amers sur les images satellitaires ou photographies aériennes, les modèles polynomiaux utilisés par le logiciel lors du géoréférencement, la position exacte du trait de côte lors de son extraction manuelle et enfin les fluctuations saisonnières du trait de côte (Dolan et al., 1991 ; Durand, 1999). Toutes ces erreurs peuvent être des facteurs de décalages supplémentaires. Il est donc important de les évaluer avant de tirer des conclusions sur la cinématique du trait de côte (Crowell et al., 1991 ; Durand,1998 et 2000).

L’incertitude liée à la marée a été négligée dans cette étude puisque :

- la date de prise de la photo aérienne 1978 n'est pas connue,

- nous ne disposons d'aucune donnée sur la marée en raison de l'absence de marégraphe opérationnel pendant la date de prise de certaines photos, 
- en plus de l'échelle des photos, les côtes sénégalaises sont des côtes micro-tidales à marnage faible (inférieur à $2 \mathrm{~m}$ ) faisant que l'influence de la marée sur la cinématique du trait de côte est faible.

Finalement, deux erreurs ont été évaluées: l'erreur liée au géoréférencement et celle liée à la digitalisation de la ligne de rivage.

Lors du géoréférencement, les erreurs liées à la résolution du scannage, à la précision des documents de référence, et au repérage des amers sur les images satellitaires ou photographies aériennes sont directement prises en compte par le programme informatique. Elles sont dans ce cas rassemblées sous le concept "erreur moyenne quadratique " ou "Root-Mean-Square Error » (RMSE) et générées directement. Dolan et al., (1991) et Durand, (2000) recommandent que cette erreur soit ramenée à \pm 1 pixel alors que Faye (2010) la considère égale à 2 pixels lorsque l'on compare deux images dont l'une est calée par rapport à l'autre. Pour notre étude, cette erreur est multipliée par la résolution de l'image pour obtenir l'incertitude liée à la correction géométrique ou géoréférencement en mètres comme l'avait fait Samat, (2007 In. Ndour, 2015). La résolution de l'image SPOT (2,5 m) utilisée comme référence lors du géoréférencement a été considérée pour le calcul de cette incertitude.

Lors de la digitalisation, la précision de la ligne extraite résulte de l'expérience du photo-interprète, de son appréciation de la ligne de référence, elle-même étant conditionnée par la résolution, l'échelle et la qualité radiométrique des images (Juigner et al., 2012). Afin de minimiser l'erreur de numérisation résultant de l'expérience du photo-interprète, les traits de côte ont été numérisés à cinq reprises pour chaque date. L'expérience de photointerprétation que cela génère amène à retenir les lignes de la dernière série, notamment lors de la superposition des lignes numérisées pour chaque date. Globalement, on obtient une précision métrique pour la ligne considérée sur des prises de vue de bonne qualité et à grande échelle comme celles de nos images. Cette erreur de digitalisation est estimée à $\pm 4 \mathrm{~m}$ (tableau 2).

Tableau 2. Estimation de la marge d'erreur globale de chaque période d'étude

\begin{tabular}{|c|c|c|c|c|c|}
\hline Supports numériques & Photo 1942 & $\begin{array}{c}\text { Photo } \\
1966\end{array}$ & $\begin{array}{c}\text { Photo } \\
1978\end{array}$ & $\begin{array}{c}\text { Photo } \\
1997\end{array}$ & $\begin{array}{c}\text { Image } \\
2011\end{array}$ \\
\hline $\begin{array}{c}\text { Incertitude de } \\
\text { géoréférencement }\end{array}$ & $\pm 0,38$ & $\pm 0,38$ & $\pm 0,33$ & \pm & $\pm 0,28$ \\
\hline $\begin{array}{c}\text { Incertitude de } \\
\text { digitalisation }\end{array}$ & \pm 4 & \pm 4 & \pm 4 & \pm 4 & \pm 4 \\
\hline $\begin{array}{c}\text { Incertitude totale en } \\
\text { mètres }\end{array}$ & $\pm 4,38$ & $\pm 4,38$ & $\pm 4,33$ & $\pm 4,28$ & $\pm 4,28$ \\
\hline $\begin{array}{c}\text { Erreur moyenne sur } \\
\text { chacune des périodes } \\
\text { considérées en m/an }\end{array}$ & $1942-1978$ & $\pm 0,24$ & \multicolumn{3}{|c|}{$1966-1997$} \\
\cline { 2 - 6 }
\end{tabular}




\section{Resultats}

L'étude par photo-interprétation de l'évolution historique de la ligne de rivage de la zone de Malibu à Malika au Nord de Dakar a été effectuée pendant la période allant de 1942 à 2011. Durant ces 69 ans, trois périodes de trente ans au minimum ont été considérées dans les analyses puisque les vitesses d'évolution obtenues sont très faibles : la première période va de 1942 à 1978 (36 ans), la deuxième période est celle de 1966 à 1997 (31 ans), la troisième et dernière période va de 1978 à 2011 (33 ans).

\section{Période 1942 à 1978 (36 ans)}

Le littoral allant de la plage de Malibu à celle de Malika est caractérisé pendant la période 1942 à 1978 par une faible prédominance des phénomènes de progradation (figure 7) matérialisée par une vitesse moyenne globale d'évolution du trait de côte de $+0,26 \mathrm{~m} / \mathrm{an}$, soit une avancée du trait de côte de $+3,24 \mathrm{~m}$ en 36 ans. Cependant, une analyse plus fine de ce segment permet de constater que cette évolution n’est pas uniforme dans l'espace (figure 7).

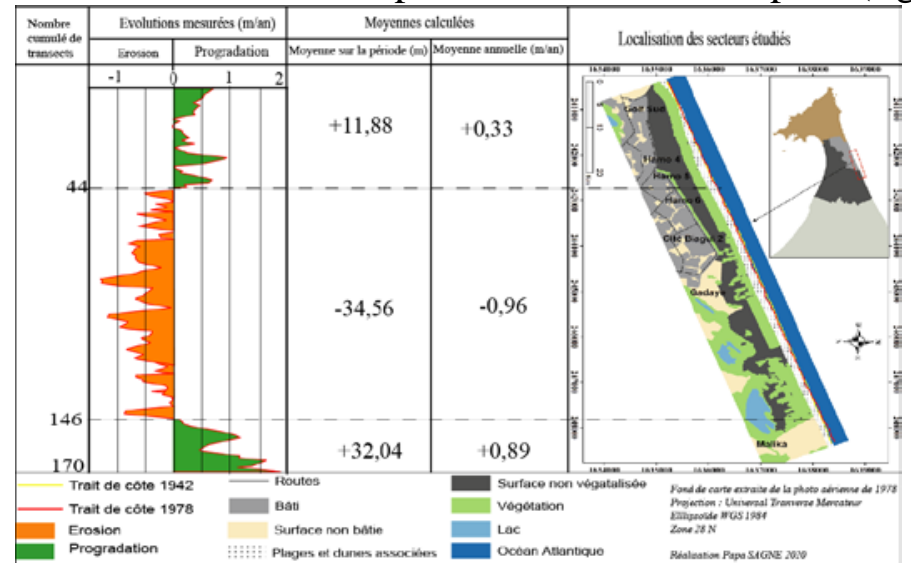

Figure 7. Evolution de la ligne de rivage du littoral allant de la plage de Malibu à celle de Malika de 1942 à 1978

Durant cette période deux secteurs en progradation et un troisième en érosion peuvent être distingués :

- pour les secteurs en progradation, le premier secteur (transects 1 à 44), situé à l'Ouest de la zone d'étude notamment entre la plage de Malibu au niveau de Golf Sud et la Cité Hamo 6 de Guédiawaye, a enregistré une avancée du trait de côte de $+11,88 \mathrm{~m}$ sur 36 ans, soit une vitesse de $+0,33 \mathrm{~m} / \mathrm{an}$. Le deuxième secteur est quant à lui situé au Nord-Est de la zone d'étude (transect 146 à transect 170) plus précisément à Malika. Il est caractérisé par une avancée du trait de côte de $+32,04 \mathrm{~m}$ sur 36 ans soit $+0,89 \mathrm{~m} / \mathrm{an}$. La vitesse des mouvements du trait de côte dans ce secteur est un peu plus importante que celle du premier secteur (+0,33 m/an) ; 
- les deux secteurs en progradation encadrent un troisième secteur en érosion de -0,96 m/an, soit -34,56 m de recul en 36 ans. Il va de la Cité Hamo 6 jusqu'à l'Ouest de Malika (transects 44 à 146) et couvre une partie de la Cité Hamo 6, toute la Cité Biagui 2 et la localité de Gadaye où cette érosion semble être dominante.

\section{Période allant de 1966 à 1997 (31 années)}

Cette période, contrairement à celle allant de 1942 à 1978, est caractérisée par un recul du trait de côte de -13,95 m en 31 ans, ce qui équivaut à une vitesse moyenne de $-0,45 \mathrm{~m} / \mathrm{an}$. Par contre, les mouvements notés sont également non uniformes dans l'espace. Sur les 170 transects considérés, les 70 premiers, c'est-à-dire de la plage de Malibu (Golf Sud) à la Cité Biagui 2 (Wakhinane Nimzatt), se situent dans un secteur en érosion avec un recul moyen de -37,51 m sur 31 années soit un taux de recul de -1,21 m/an (figure 8). L’érosion devient moins intense vers la Cité Biagui 2. Le reste de la zone d'étude (qui va de la Cité Biagui 2 à Malika) est en progradation avec une avancée de +23,56 m. La vitesse moyenne d'évolution correspondante est de $+0,76 \mathrm{~m} / \mathrm{an}$. Certaines particularités peuvent être notées pendant cette période comparée à la précédente :

- de la plage de Malibu (Golf Sud) à la cité Biagui 2 (du premier transect au $70^{\text {ème }}$ transect) où prédominaient des mouvements accrétionnels lors de la période 1942-1978, une inversion de la tendance évolutive est notée entre 1966 et 1997 avec une prédominance des mouvements érosifs occasionnant un recul du trait de côte avec une vitesse de $-1,21 \mathrm{~m} / \mathrm{an}$;

- du transect 70 au transect 146 (de la cité Biagui 2 à Malika) où il y’avait une prédominance des mouvements érosifs entre 1942 et 1978, l'évolution est en faveur d'une progradation avec une vitesse de +0,76 m/an de 1966 à 1997 ; -la plage de Malika a, par contre, connu une diminution du taux d'avancée du trait de côte. Aucune situation d'érosion n'est encore notée à ce niveau.

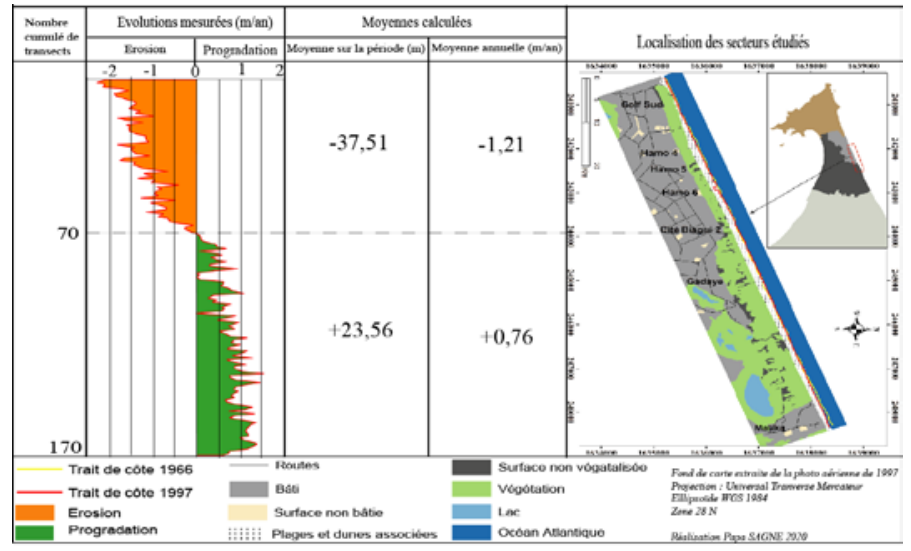

Figure 8. Evolution de la ligne de rivage du littoral allant de la plage de Malibu à celle de Malika de 1966 à 1997 


\section{Période de 1978 à 2011 (33 ans)}

Pendant cette période, l'érosion précédemment notée de 1966 à 1997 se poursuit mais avec une forte augmentation de la vitesse de recul du trait de côte. Celle-ci, initialement de $-0,45 \mathrm{~m} /$ an entre 1966 et 1997 passe à $-1,58$ $\mathrm{m} /$ an entre 1978 et 2011 soit un recul de $-52,14 \mathrm{~m}$ en 33 ans. Une analyse plus fine montre que le recul du trait de côte noté pendant la période 1966-1997 dans le secteur allant de la plage Malibu à la Cité Biagui 2 (transects 1 à 70) se poursuit entre 1978 et 2011. Cependant, la vitesse d'érosion a diminué pendant cette dernière période ; elle passe de -1,21 m/an entre 1966 et 1997 à $-0,73 \mathrm{~m} / \mathrm{an}$ entre 1978 et 2011 ; ce qui correspond à un recul du trait de côte de $-24,49$ m en 33 ans. Le secteur de Malika, situé entre les transects 145 et 170 initialement en progradation de 23,56 m pendant la période 1966-1997, est marqué pendant entre 1978 et 2011 par une érosion de -39,6 m. La vitesse de recul de $-1,28 \mathrm{~m} / \mathrm{an}$, fait que le secteur de Malika a enregistré le plus grand taux de recul pendant cette période. Les transects 70 à 145 (Cité Biagui 2 à Gadaye) se situent quant à eux dans un secteur en progradation avec une avancée de $+14,19$ m correspondant à un taux moyen de $+0,43 \mathrm{~m} /$ an (figure 9).

Comparée à la précédente période, celle de 1978 à 2011 est caractérisée par :

- une réduction des taux d'érosion au niveau du secteur allant de la plage de Malibu à la Cité Biagui 2 ;

- une inversion de sa tendance évolutive s’observe à la plage de Malika avec un recul de son trait de côte entre 1978 et 2011.

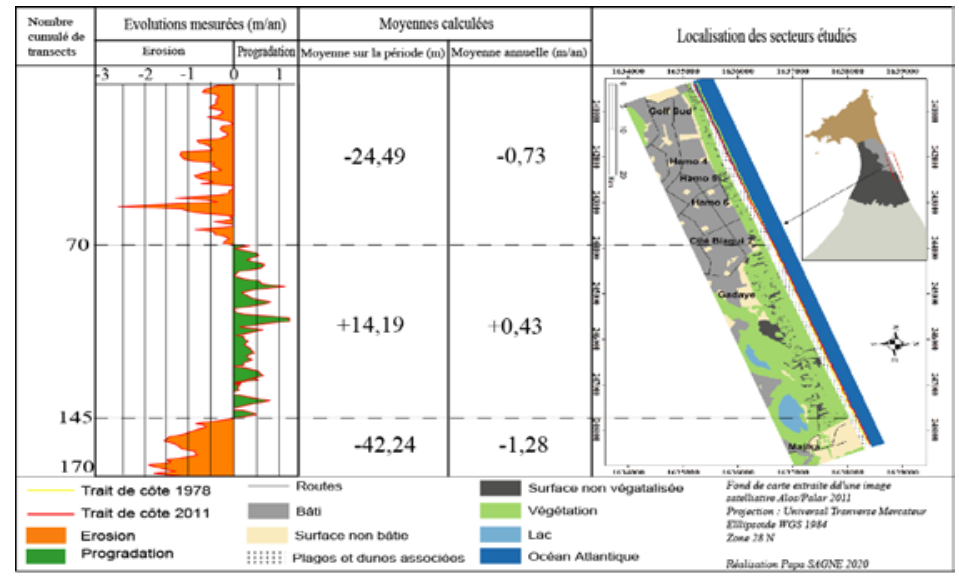

Figure 9. Evolution de la ligne de rivage du littoral allant de la plage de Malibu à celle de Malika de 1978 à 2011

La période d'étude : 1942 à 2011

L'analyse des taux d'évolution de la ligne de rivage obtenus par la méthode de la régression linéaire de 1942 à 2011 laisse apparaitre une 
évolution du trait de côte en faveur d'une érosion avec une vitesse moyenne globale de $-0,15 \mathrm{~m} / \mathrm{an}$. Cependant, cette érosion n'est pas uniforme sur toute la zone. Ainsi trois secteurs selon le sens d'évolution du trait de côte et l'ampleur des mouvements sont nettement distincts.

Le premier secteur est en érosion et se situe à l'Ouest depuis la plage de Malibu jusqu'à la Cité Biagui 2 (transects 1 à 90). La vitesse moyenne de recul du trait de côte est de $-0,22 \mathrm{~m} / \mathrm{an}$. On note que cette érosion devient plus intense de la Cité Biagui 2 à la plage de Malibu. Le troisième secteur est aussi en érosion et se situe dans la zone de Malika (transects 144 à 170). Il présente une vitesse d'évolution du trait de côte de $-0,04 \mathrm{~m} / \mathrm{an}$, très faible comparée à celle du premier secteur. Ces secteurs encadrent une autre qui est quant à lui en progradation. Il couvre toute la zone de Gadaye et va du Nord-Est de la cité Biagui 2 jusqu'à l'Ouest de Malika (transects 90 à 144). Le taux d'évolution est de $+0,11 \mathrm{~m} /$ an (figure 10 ).

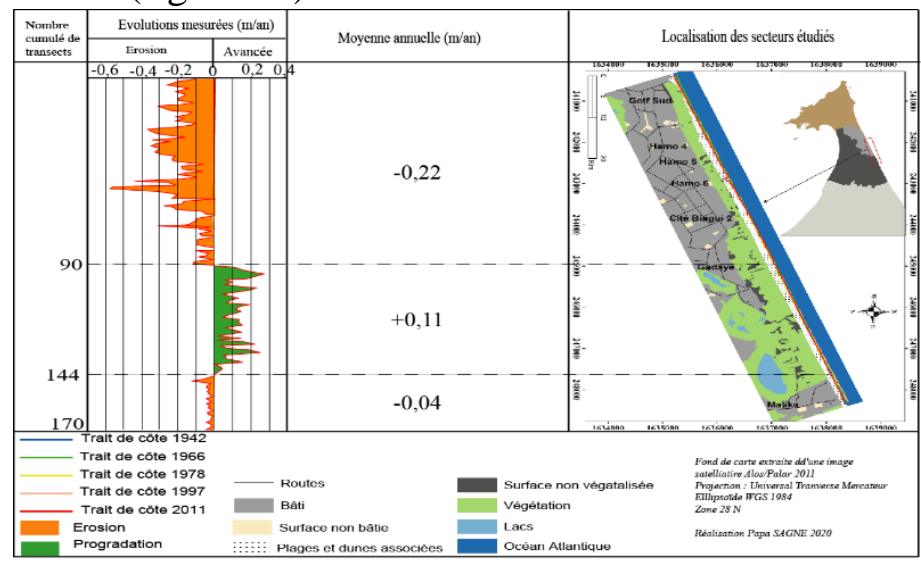

Figure 10. Evolution de la ligne de rivage du littoral allant de la plage de Malibu à celle de Malika de 1942 à 2011

\section{Discussions}

L'analyse de l'évolution de la ligne de rivage durant la période allant 1942 à 2011 par la méthode de la régression linéaire montre une prédominance des mouvements de recul du trait avec une vitesse moyenne globale de $-0,15$ $\mathrm{m} / \mathrm{an}$. Ce recul n'est cependant pas uniforme le long de la zone d'étude et durant toute la période d'étude indiquant une certaine variabilité spatiotemporelle des mouvements du trait de côte. En effet, la plage de Malika avait enregistré une avancée du trait de côte avec une vitesse de $+0,89 \mathrm{~m} / \mathrm{an}$ entre 1942 et 1978, puis une inversion de la tendance évolutive marquée par un recul du trait de côte de $-1,28 \mathrm{~m} /$ an entre 1978 et 2011. De la même manière, la zone de Malibu à Hamo 6, initialement en progradation de +0,33 m/an entre 1942 et 1978, s'est retrouvée dans une situation d'érosion entre 1978 et 2011 avec un taux de recul du trait de côte de $-0,73 \mathrm{~m} / \mathrm{an}$. Une alternance des 
mouvements de recul et de progradation est aussi notée dans la zone de Cité Biagui 2 et surtout à Gadaye. Cependant, une prédominance des mouvements de progradation serait à l'origine de la tendance accrétionnelle notée à Gadaye par la méthode de la régression linéaire. Cette variabilité spatio-temporelle des mouvements du trait de côte aurait traduit une variation de l'énergie des houles d'un secteur à un autre et d'une période à une autre comme l'avait noté Fall (2004) à Yoff. Les variations spatiales de la dynamique du trait de côte pendant une même période pourraient également être liées à des différences sectorielles de la bathymétrie puisque celle-ci à une forte influence sur le déferlement des houles et vagues. Ceci fait qu'une étude bathymétrique semble être nécessaire pour mieux comprendre la dynamique de ce milieu.

L'étude de l'évolution de l'occupation du sol montre que les installations humaines absentes dans la bande côtière de la zone d'étude jusqu'en 1966, ont été notées en 1978 et se sont multipliées depuis cette date jusqu'en 2011 en se rapprochant davantage du rivage (figures 11, 12, 13, 14 et 15).

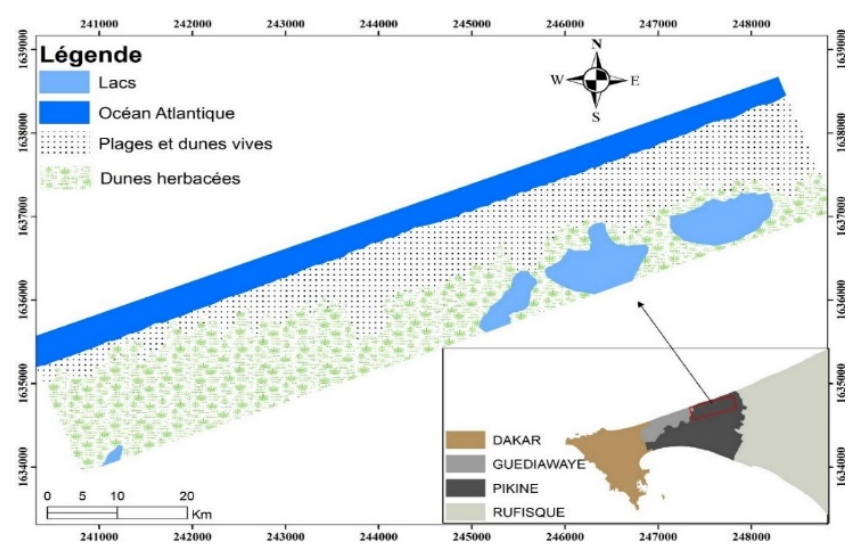

Figure 11. Occupation du sol de la zone nord de Dakar en 1942

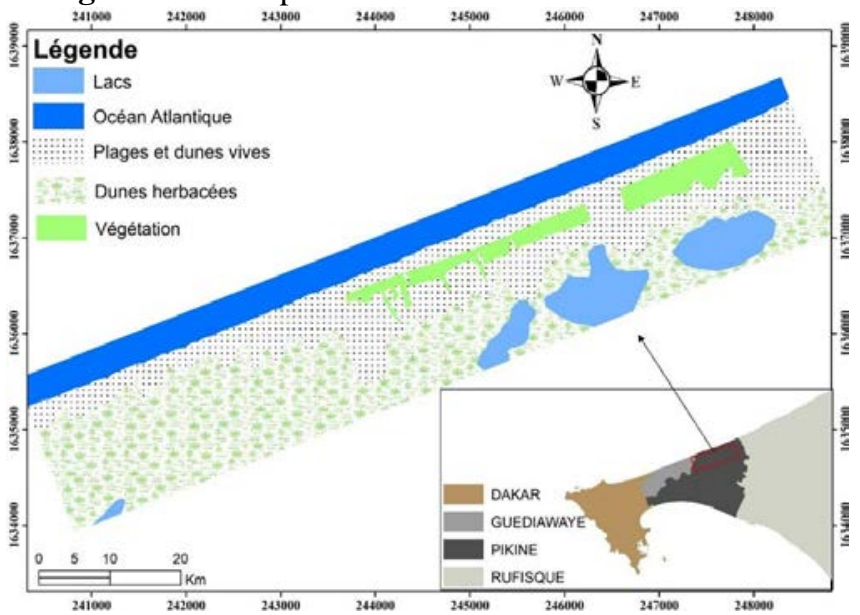

Figure12. Occupation du sol de la zone nord de Dakar en 1966 


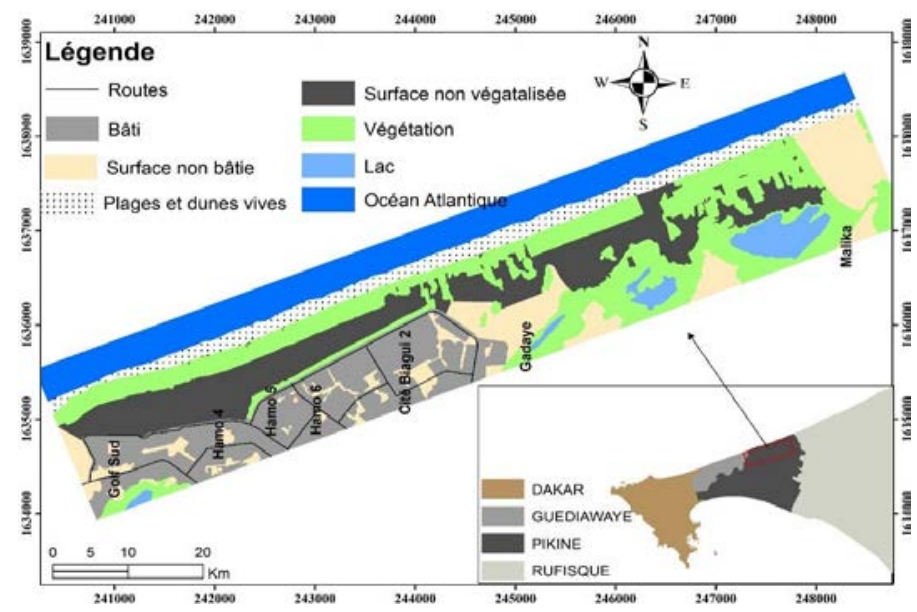

Figure 13. Occupation du sol de la zone nord de Dakar en 1978

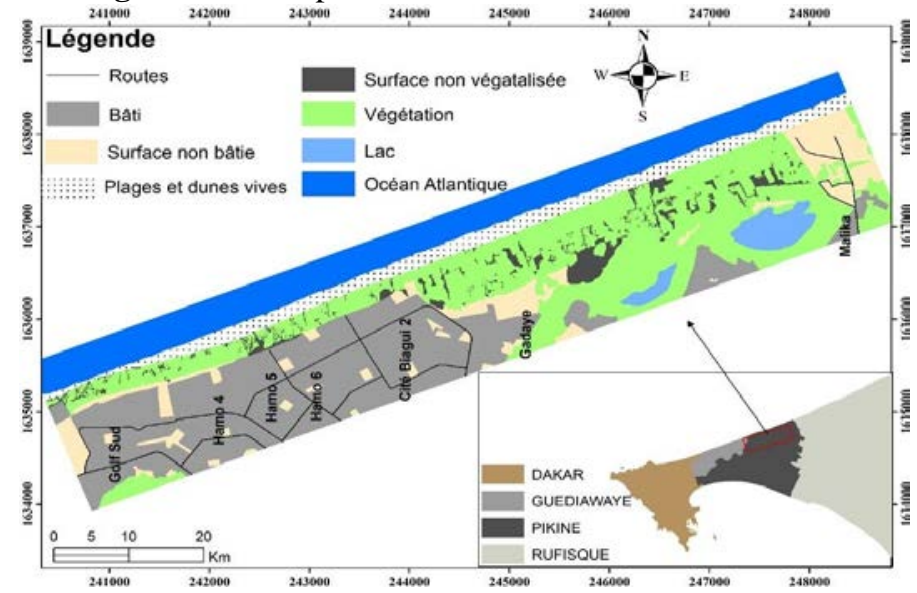

Figure 14. Occupation du sol de la zone nord de Dakar en 1997

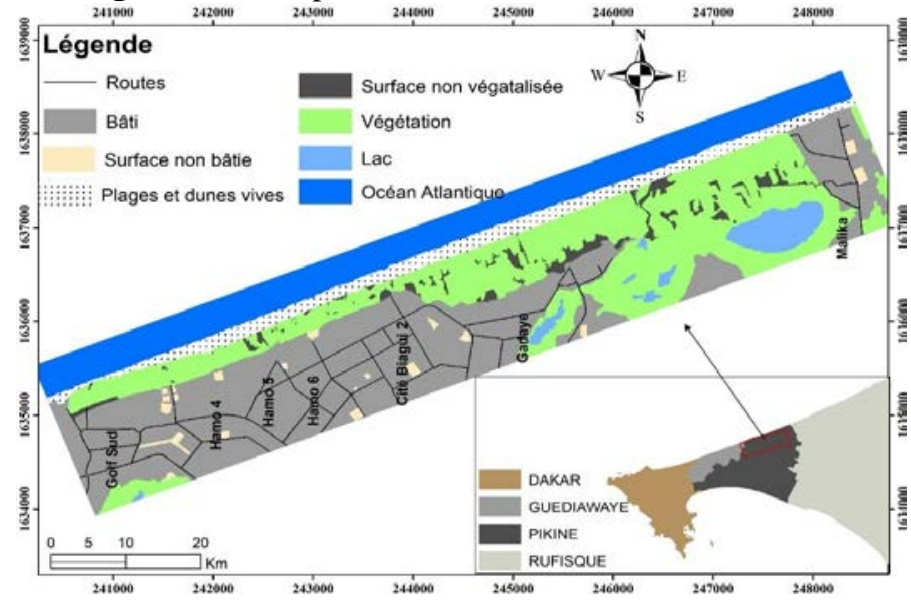

Figure 15. Occupation du sol de la zone nord de Dakar en 2011 
Cette urbanisation croissante se traduit par une forte demande en matériaux de construction notamment en sable marin. Des prélèvements frauduleux de sables qui se font soit au niveau des dunes soit directement sur la haute plage ou même sur l'estran ont été signalés à Malika (Ndao, 2012). Ils peuvent dans ce cas contribuer à accroitre le déficit sédimentaire des plages et rendre la zone plus sensible à l'action des fortes houles. Ce fait peut, dans le long terme, affecter la dynamique du trait de côte dans certains endroits. Ces extractions de sables peuvent donc être considérées comme une cause probable du recul du trait de côte à ce niveau. Cependant, la carte de l'occupation du sol de 2011 montre qu'il existe toujours une certaine distance entre ces installations humaines et le rivage en raison de la bande de filaos notée dans cette partie. Ceci exclue jusque-là des actions humaines directes, à l'exception des extractions de sables signalées en haut, sur les processus d'évolution de cette côte. Ainsi, jusqu'en 2011, les véritables causes de l'évolution côtière dans cette partie du littoral de Dakar seraient probablement dues aux extractions frauduleuses de sables côtiers et à l'action des agents hydrodynamiques particulièrement les houles du Nord-Ouest, facteurs prépondérants qui gouvernent les mouvements des sédiments (Joire, 1947) puisque la zone est protégée des houles du Sud-Ouest par la configuration géomorphologique de la région de Dakar et que l'effet de la marée est très faible. De la même façon Sall (1982) considère la houle du Nord-Ouest comme le principal agent dynamique déterminant l'évolution de la côte nord de Dakar. Néanmoins, la pression humaine commence à se faire mieux sentir actuellement sur cette partie nord du littoral de Dakar avec l'extension de la Voie de Dégagement Nord qui facilite le déplacement des personnes et biens vers cette partie. A cela s'ajoute la forte croissance démographique notée ces dernières décennies. Tous ces facteurs font que les filaos commencent à être abattus au profit des constructions humaines surtout entre 2018 et 2019. Les conséquences de la destruction de ces filaos peuvent être alarmantes. En effet, Bakhoum et al. (2018) avait montré que la forte vulnérabilité physique de la Grande Côte à l'élévation du niveau de la mer dans ce contexte de changement climatique serait, en partie, liée à des coupes de filaos. En effet, au niveau des zones littorales, c'est la végétation comme les filaos qui freine le vent, piège et stabilise le sable en mouvement (Paskoff, 1993) pour former les dunes littorales. Ces dunes sont d'une grande importance pour ces zones. Ainsi, toute destruction des filaos pour faire place à des constructions pourrait entrainer une réduction du stock sédimentaire dunaire voire une disparition des dunes et la suppression de leurs rôles de protection naturelle contre l'érosion côtière surtout dans ce contexte actuel de changement climatique. Il est donc impératif que cette bande de filaos soit protégée.

En comparant les résultats de cette étude avec ceux trouvés dans d'autres zones, les observations suivantes sont faites. La vitesse maximale de 
recul de trait de côte qui est de -1,28 m/an et enregistrée entre 1978 et 2011 à Malika est légèrement plus faible que celle trouvée à Yoff située dans la même zone de même que celle trouvée dans d'autres zones de Dakar, même si les méthodes d'étude ou les périodes considérés sont quelque part différentes. En effet, au niveau du littoral de Yoff, les taux varient entre -0,7 et -1,7 m/an (Fall, 2014). A Rufisque, Ndour (2015) a trouvé par la même méthode que la présente étude des taux d'évolution atteignant $-1,51 \mathrm{~m} / \mathrm{an}$. Au niveau des littoraux de Bargny, Ngoudou, de Bargny Guedj et de Siendou au Sud de Dakar, les vitesses de recul du trait de côte sont respectivement de $-1,58 \mathrm{~m} / \mathrm{an}$ ; -1,04 m/an et -0,97 m/an entre 1927 et 1997 (Faye, 2010).

Ces tendances évolutives du trait de côte indiquent que les côtes nord et sud de Dakar sont toutes en érosion. Plusieurs raisons peuvent expliquer les différences notées. Au niveau du littoral de Yoff, en plus de l'absence de dunes actives qui devraient stabiliser et alimenter la plage en sédiments, les activités humaines participent à l'augmentation de la sensibilité de cette côte à l'action des fortes houles responsables en grande partie de son érosion (Fall, 2004). Cette situation est comparable à celle de Rufisque mais qui est plutôt protégée des houles du Nord-Ouest par la configuration de la Presqu'île du Cap Vert (Ndour, 2015). L'autre particularité de cette zone est que les structures de protection telles que les épis et murs de protection exercent une influence notoire sur l'évolution du trait de côte (Ndour, 2015) alors qu'à Yoff ce sont plutôt les extractions de sables associées à l'occupation anarchique de l'arrière plage qui sont déterminantes. A Bargny, ce sont aussi les extractions frauduleuses de sables et l'occupation anarchique qui déstabilisent le littoral (Faye, 2010). La zone de Malibu à Malika est quant à elle caractérisée par un stock sédimentaire puissant en raison de la présence de massif dunaire important. Celui-ci alimente les plages en sédiments et permet de renouveler leur stock sédimentaire pendant les périodes de fortes houles érosives. Ce fait permet de ralentir le recul du trait de côte grâce à une alternance de mouvements d'érosion majoritairement liés aux houles fortes et de mouvements d'accumulation. Toutefois des mouvements d'érosion dominants dus aux extractions de sables et à l'action des houles du Nord-Ouest seraient à l’origine de ce recul.

\section{Conclusion}

L'étude par photo-interprétation de l'évolution historique de la ligne de rivage de la zone de Malibu à Malika au Nord de Dakar montre par la méthode de la régression linéaire que la période allant de 1942 à 2011 est caractérisée par des taux d'érosion variant entre -0,04 m/an et -0,22 m/an et un petit secteur en accrétion situé entre Gadaye et Malika avec une vitesse de $+0,11 \mathrm{~m} / \mathrm{an}$. Ceci donne une vitesse moyenne globale de $-0,15 \mathrm{~m} / \mathrm{an}$. L'analyse de l'évolution de l'occupation du sol montre une urbanisation de plus en plus 
importante dans la zone entre 1942 et 2011. Cependant, l'impact de cette urbanisation sur les fluctuations de la ligne de rivage n'est pas encore véritablement établi, ce qui confirme bien que les facteurs qui déterminent le plus l'évolution de ce segment littoral sont les houles du Nord-ouest auxquelles s'ajoutent les extractions de sables. Toutefois, avec l'extension de la Voie de Dégagement Nord, les coupes de filaos au profit des constructions humaines, il pourrait se produire une certaine influence des activités humaines dans le fonctionnement de ces plages en favorisant la rupture des échanges sédimentaires entre les dunes et la plage par immobilisation des sédiments dunaires sur lesquelles s'effectuent les constructions. Les conséquences peuvent être l'accélération de la vitesse de recul du trait de côte et/ou la généralisation de l'érosion dans cette partie.

Cette étude mérite donc d'être approfondie par l'utilisation de données météo-marins et de techniques plus modernes telles que le DGPS ou le LIDAR et les drones afin de produire des modèles d'évolution plus précis de toute la bande côtière. Une étude bathymétrique permettrait également de mieux expliquer les variations spatiales du trait de côte pendant une même période. Il serait aussi important d'effectuer une étude de vulnérabilité physique et socio-économique et de produire des modèles d'évolution future qui peuvent permettre de mieux prévoir les conséquences de la pression humaine sur l'évolution du rivage.

\section{Remerciement}

Les auteurs de cet article s'inclinent très respectueusement devant la mémoire du feu Professeur Isabelle Niang qui leur a initié à la recherche. Ils lui rendent un vibrant hommage et lui souhaitent que la terre lui soit légère.

\section{References:}

1. ANSD Agence national de la Statistique et de la démographie (2014). Résumé des chapitres du rapport définitif RGPHAE, Dakar, Sénégal.

2. Bâ K. (2013). Apport de la télédétection et des SIG dans l'étude de l'évolution de la langue de Barbarie et de l'estuaire du fleuve Sénégal. Thèse de Doctorat, Université Cheikh Anta Diop de Dakar. 212 pp, 106 fig, 30 tab, 10 photos.

3. Bakhoum P. W., Niang I., Sambou B., et Diaw A. T. (2018). Physical vulnerability of Dakar region facing sea levels rising $s$ in the context of climate change. Environmental and Water Sciences, Public Health Territorial Intelligence Journal, vol. 2, no 3, p. 1126.

4. Balle G. R. A., Ahouansou D. M. M., Sintondji L. C.,Agbossou E. K. (2021). Analyse des tendances du littoral à court et à long terme de la côte sud-ouest du Bénin. Journal of Coastal Research, volume 37 numéro 2, 316-325. 
5. Bernabeu A. M., Medina R. et Vidal C. (2003). A morphological model of the beach profile integrating wave and tidal influences. Marine. Geology, vol. 197, no 1, p. 95116.

6. Bird E.C.F. (1985). Coastline changes. A global review. John Wiley \& Sons, Chichester, New York, Brisbane, Toronto, Singapore, 219 p.

7. Crowell M., Leathermann S. P., and Buckley M. K. (1991). Historical shoreline change: error analysis and mapping accuracy. Journal of Coastal Research, 7(3), 839-852.

8. Crowell M., Leatherman S., Buckley M.K., (1993). Shoreline change rate analysis: long term versus short term data. Shore and Beach, vol. $61, n^{\circ} 2$, p. $13-20$.

9. CSE Centre de Suivi Ecologie (2010). Rapport sur l'état de l'environnement au Sénégal

10. Daniel F., Hubaud M.O. (1996). Érosion côtière des systèmes littoraux sableux. ODEM, $21 \mathrm{p}$.

11. Diaw A.T. (1984). Morphométrie du littoral sénégalais et gambien. Notes Africaines, Dakar, 183, 58-63, 5 fig., 1 tab.

12. Dolan R., Hayden B. P., Heywood J. (1978). A new photogrammetric method for determining shoreline erosion. Coastal Engineering, 2, 21 $-39$

13. Dolan R., Hayden B. P., May P. and May S. (1980). The reliability of shoreline change measurements from aerial photographs. Shore and Beach, 48(4), 22-29.

14. Dolan, R., Fenster, M.S., Holme, S. J. (1991). Temporal analysis of shoreline recession and accretion. Journal of Coastal Research, 7, 723744.

15. Douglas B.C. and Crowell M. (2000). Long term shoreline prediction and error propagation. Journal of Coastal Research, 16, 1, 145-152.

16. Durand P. (1998). Cinématique d'un littoral sableux à partir de photographies aériennes et de cartes topographiques. Exemple du littoral d'Argelès-plage à Saint-Cyprien (Roussillon, France). Géomorphologie : relief, processus, environnement, 2, 155-166.

17. Durand P. (1999). L'évolution des plages de l'ouest du golfe du Lion au XXe siècle. Cinématique du trait de côte, dynamique sédimentaire, analyse prévisionnelle. Thèse de Doctorat, Université Lyon II, 2, $461 \mathrm{p}$.

18. Durand P. (2000). Approche méthodologique pour l'analyse de l'évolution des littoraux sableux par photo-interprétation. Photointerprétation, 38(1-2), 3-18.

19. Emmanuel B. (2017). Etude des dynamiques du trait de côte de la région Bretagne à différentes échelles spatio-temporelles. Géographie. 
Université de Bretagne Occidentale — Brest. Français. <NNT : 2017BRES0010>.

20. Fall B. (2004). Le littoral de Yoff : évolution morphosédimentaire et changements à long terme de la ligne de rivage. Thèse 3ème cycle, Université Cheikh Anta Diop de Dakar. 165 pp, 41 tab, 70 fig, 8 photos.

21. Faye I. (2010). Dynamique du trait de côte sur les littoraux sableux de la Mauritanie à la Guinée-Bissau (Afrique de l'Ouest) : Approches régionale et locale par photo-interprétation, traitement d'images et analyse de cartes anciennes. Thèse de Doctorat, Université de Bretagne Occidentale, 393 p.

22. Fenster M. S., Dolan R., Elder J. F. (1993). A new method for predicting shoreline positions from historical data. Journal of Coastal Research, vol. 9, n ${ }^{\circ}$, p. 147 - 171.

23. Fletcher C.H., Rooney J.J., Barbee M., LIM S.C., and Richmond B. (2003). Mapping shoreline change using digital orthophotogrammetry on Maui, Hawaii. Journal of Coastal, Research, Special Issue $n^{\circ} 38$, pp 106 - 124.

24. Hénaff A., Lageat Y., Hallégouët B., Jabbar M., Delliou N.et Diard M. (2015). Évolutions des accumulations littorales et relations avec les dynamiques d'avant-plage dans l'archipel des Glénan (Sud-Finistère, France), Géomorphologie : relief, processus, environnement, vol. 21$\mathrm{n}^{\circ}$ 4, DOI : 10.4000/geomorphologie.11195

25. Grenier A., Dubois J.M. (1990). Evolution littorale récente par télédétection : synthèse méthodologique. Photo-Interprétation, vol 29, $\mathrm{n}^{\circ} 6$, pp. 3-7

26. Ibe A.C., Quelennec R.E. (1989). Méthodologie d'inventaire et de contrôle de l'érosion côtière dans la région de l'Afrique de l'Ouest et du Centre. Rapports et Etudes mers régionales, PNUE, Nairobi, 107 p.

27. JOIRE J. (1947). Amas de coquillage du littoral sénégalais dans la banlieue de Saint-Louis. Bulletin de l'Institut Français d'Afrique noire, Tome 9, n 1-4, p. 170 - 340.

28. Juigner M. (2012). Cinématique d'un trait de côte sableux en Vendée entre 1920 et 2010, Méthode et analyse, Géolittomer CNRSUMR 6554 LETG, Université de Nantes, 13p.

29. Juigner M., Robin M., Fattal P., Maanan M., Le Guern C., Gouguet L., Baudouin V., Debaine F. (2012). Cinématique d'un trait de côte sableux en Vendée entre 1920 et 2010: méthode et analyse, https://www.researchgate.net/publication/278624019.

30. Konan K. E., Abe J., Aka K., Neumeier U., Nyssen J., et Ozer A. (2016). Impacts des houles exceptionnelles sur le littoral ivoirien du 
Golfe de Guinée. Géomorphologie Relief Process. Environ., vol. 22, no vol. $22-\mathrm{n}^{\circ}$ 1, p. 105-120, doi: 10.4000/geomorphologie.11241.

31. Le Berre I., Henaff A., Devogele T.,MascreT A., et Wenzel F. (2016). SPOT5 : un outil pertinent pour le suivi du trait de côte ? Norois, DOI : 10.4000/norois.378.

32. Mall I. (2016). Evaluation des ressources en eau dans le Sénégal oriental : apports des outils géochimiques, géostatistiques, de la télédétection et des SIG. Thèse de Doctorat Unique, Université Cheikh Anta DIOP, 202 pp. 15 photos, 27 tab. 92 fig.

33. Moore L. J. (2000). Shoreline mapping techniques. Journal of Coastal Research, 16, p. 111 - 124.

34. Mugica J., Mallet C., Hennequin V. (2008). Etude de l'évolution géomorphologique récente de la côte de sableuse aquitaine. BRGM/RP-56874-FR, 29 ill., 9 ann., 165 pp.

35. Ndao M. (2012). Dynamiques et gestions environnementales de 1970 à 2010 des zones humides au Sénégal : étude de l'occupation du sol par télédétection des Niayes, Djidakh, Thiaroye Kao (à Dakar), Mboro (à Thiès) et Saint-Louis. Thèse de Doctorat, Université de Toulouse. $371 \mathrm{pp}, 71$ photos, 57 tab, 56 fig.

36. Ndour A. (2015). Evolution morphosédimentaire et impacts des ouvrages de protection sur le littoral de Rufisque, Petite Côte, Sénégal. Thèse de Doctorat, Université Cheikh Anta Diop de Dakar. 239 pp, 43 tab, 93 fig, 20 photos.

37. Niang-Diop I. (1995). L'érosion côtière sur la Petite-Côte du Sénégal à partir de l'exemple de Rufisque. Passé - Présent - Futur. Thèse Université, Angers, tome 1, 318 pp, 112 fig., 47 tab

38. PANA Plan d'Action National d'Adaptation (2006). Rapport du Ministère de l'Environnement et de la Protection de la Nature 84 pp., 10 figures, 19 tableaux

39. Paskoff R. (1993). Côtes en danger. Masson, Paris, 250 pp, 50 fig.

40. Pinot J. P. (1998). Gestion du littoral. Tome I- littoraux tempérés : côtes rocheuses et sableuses. Institut océanographique, Paris, 399 pp, 222 fig

41. Provencher L., Dubois J.M.M. (2007). Précis de télédétection. Volume 4. Méthodes de photo-interprétation et d'interprétation d'image. Presses de l'université du Québec / Agence universitaire de la Francophonie, Québec, 468 p.

42. Roger J., Banton O., Barusseau J.P., Castaigne P., Comte J-C., Duvail C., Nehlig P., Noël B.J., Serrano O., Traviy. (2009). Notice explicative de la cartographie multi-couches à 1/50 000 et 1/20 000 de la zone d'activité du Cap-Vert. Ministère des Mines, de l'Industrie et des PME, Direction des Mines et de la Géologie, Dakar, 245 pp. 
43. Sabatier F. and Suanez S. (2003). Evolution of the Rhône delta coast since the end of the 19th century. Géomorphologie: relief, processus, environnement, 4, 283-300.

44. Sagne P., Fall B., Bâ K. et Sow E., H. (2019). Morphosedimentary evolution of Mamelles and Ouakam beaches (Dakar, Senegal); Environmental and Water Sciences, Public Health Territorial Intelligence Journal, Volume 3 Issue 4,

45. Sagne P., Fall B., Bâ K., Faye G., Sow E., H. et Niang I. (2020). Impacts of the storm surges on sandy beaches: example of northern coast of Dakar (Senegal); Environmental and Water Sciences, Public Health Territorial Intelligence Journal, Volume 4 Issue 1,

46. Sall M. (1982). Dynamique et morphodynamique actuelles au Sénégal occidental. Thèse d'Etat Lettres, Université Strasbourg, 604 pp., 158 fig., $21 \mathrm{pl}$.

47. SRSD Service Régional de la Statistique et de la Démographie de Dakar (2015). Situation économique et social de la Région de Dakar en 2013.

48. Shoshany M. and Degani A. (1992). Shoreline detection by digital image processing of aerial photography. Journal of Coastal Research, 8 (1), Fort Lauderdale (Florida), pp. 29-34

49. Stéphanain A., Balouin Y., Belon R., et Bodéré G. (2011). Etude complémentaire sur le littoral de la plaine Orientale de Corse - état des connaissances sur les impacts des tempêtes sur le littoral. Rapport final du BRGM RP- 59058-FR 137pp., 64 ill., 4 ann.

50. Suanez S. et Cariolet J.-M. (2010). L'action des tempêtes sur l'érosion des dunes : les enseignements de la tempête du 10 mars 2008 », Norois Environ. Aménage. Société, no 215, p. 7799, doi: 10.4000/norois.3212.

51. Suanez S. et Simon B. (1997). Utilisation de l'analyse diachronique dans l'étude de l'évolution du littoral oriental du delta du Rhône (France, Sud-Est). Photo-Interprétation, 3-4, 147-154.

52. Thieler E.R., et Danforth W.W. (1994). Historical shoreline mapping (I): Improving techniques and reducing positioning errors. Journal of Coastal Research, vol. 10, n³, p. 549 - 563.

53. Thieler E.R., Himmelstoss E., et Miller T. (2005). User guide and tutorial for the Digital shoreline analysis system (DSAS) version 3.2,USGS Open-File Report 2005-1304, 33p.

54. Thieler E.R., Himmelstoss E. A., Zichichi J. L., et Ergul. A. (2009). Digital Shorline Amalysis Sytem (DSAS) version 4.0- An ArcGIS extension for calculating shoreline change : U.S. Geological Survey Open-File Report 2008-1278. 
55. Thior M., Sané T., Sy O., Descroix L., Ba B. D., Soly B., et Mendy V. (2019). Analyse Spatiale de l'évolution du Trait de Côte autour de l'embouchure du Fleuve Casamance (Sénégal) de 1968 à 2017, à Partir de l'outil DSAS. European Scientific Journal, Vol.15, No.9

56. Young A.P., Olsen, M.J., Driscoll, N., Flick R.E., Gutierrez R., Guza R.T., Johnstone E. et Kuester F., 2010. Comparison of airborne and terrestrial lidar estimates of seacliff erosion in southern California. Photogramm. Eng. Remote Sens. 76, 421-427. 